\title{
Insertion Des Jeunes À Risque À Travers Les Opportunités D'activités Économiques Offertes Dans Le Secteur Des Transports En Commun À Abidjan
}

\author{
Dr. Crizoa Hermann \\ Maître-assistant à l'UFR Criminologie (UFHB), Côte d'Ivoire
}

Doi:10.19044/esj.2018.v14n32p102 URL:http://dx.doi.org/10.19044/esj.2018.v14n32p102

\begin{abstract}
The study aims to show that the public transport sector in Abidjan, constitutes a pool of jobs likely to allow the social integration of young people at risk of committing crimes and offenses. To achieve this goal, 207 people were interviewed empirically from a judgment sample through municipalities in the city of Abidjan. The data collected were analysed and processed qualitatively and quantitatively. The results of the survey reveal that the Abidjan public transport environment, essentially perceived as a niche for the development of urban insecurity, enables the social integration of many young people at risk by the opportunities for economic activities that are available to them without qualification.
\end{abstract}

Keywords: Economic activity, employment pool, insecurity, integration, youth at risk, public transport

\section{Resumé}

L'étude a pour objectif de montrer que le secteur des transports en commun à Abidjan, constitue un bassin d'emplois susceptible de permettre l'insertion sociale des jeunes à risque de commettre des crimes et délits. Pour atteindre cet objectif, 207 personnes ont été interrogés de façon empirique à partir d'un échantillonnage par jugement à travers des communes de la ville d'Abidjan. Les données recueillies ont été analysées et traitées au plan qualitatif et quantitatif. Les résultats de l'enquête révèlent que le milieu des transports en commun abidjanais, est essentiellement perçu comme une niche de développement de l'insécurité urbaine, permet l'insertion sociale de nombreux jeunes à risque par les opportunités d'activités économiques qui leurs sont offertes sans qualification.

Mots-Cles: Activité économique, bassin d'emploi, insécurité, insertion, jeunes à risque, transports en commun 


\section{INTRODUCTION}

L'insécurité dans les transports en commun est l'objet d'une construction médiatique qui la place au-devant de la scène. Un peu partout dans le monde, les transports en commun sont présentés, autant par la presse que des travaux de recherche, comme des espaces de violence et de délinquance.

En France, le CERTU (2004) note depuis une quinzaine d'année, une aggravation des situations de violence dans les transports publics. Selon l'ONDRP (2016), près d'un tiers (1/3) des agressions et atteintes personnelles (insultes, menaces, violences ou vols) déclarées par les victimes dans les lieux publics ont lieu dans les transports en commun. Cette situation est la même en Belgique où elle expose particulièrement les usagers et le personnel des transports en commun aux risques d'agressions et de violences (Toumpsin, 2006).

À la suite de l'Europe, le continent américain n'est pas en reste. À Montréal (Canada), Ouimet \& Tremblay (2001) révèlent que l'insécurité dans les espaces publics est cinq (5) fois plus élevés dans le métro que dans les autres espaces publics. Garcia (2006), fait également remarquer qu'en Amérique Latine, l'insécurité dans les transports en commun est inquiétante et est surtout liée aux enlèvements dans les taxis.

Cette forme de criminalité est aussi présente en Afrique (Lombard \& Ninot, 2002). À Abidjan (Côte d'Ivoire) notamment, la violence dans les transports en commun sévit avec acuité (Crizoa (2010, 2013, 2014a, 2014b) et Crizoa et al. 2017).

De ce qui précède, l'on peut dire que l'insécurité dans les transports en commun s'observe dans la plupart des sociétés contemporaines. Les images que véhiculent les médias et les systèmes politiques sont souvent dégradantes. Mais des tendances positives permettent de penser que ce secteur d'activité est une porte d'entrée honorable pour des personnes à risque, contraintes d'améliorer leurs conditions de vie. En Côte d'Ivoire par exemple, le secteur artisanal des transports en commun assuré par les "gbakas"" et les "wôrôwôrô" ${ }^{6 "}$ à Abidjan, se révèle attractif pour de nombreux jeunes à risque. Ce secteur présente une forte capacité à pourvoir un nombre significatif d'emplois économiquement rentable et socialement acceptable pour des individus sans qualification et particulièrement vulnérables. Ce modèle d'insertion par l'activité économique pourrait d'ailleurs représenter la solution au problème de la double logique d'insertion sociale et professionnelle des jeunes sans qualification (Duchène \& Poplimont, 2013). Pour ces jeunes d'ailleurs, l'emploi est pensé comme pourvoyeur de revenus et le travail est vu comme

\footnotetext{
${ }^{5}$ Ce sont des minibus de 14 à 39 places, qui assurent la desserte de la périphérie d'Abidjan à partir de la commune d'»Adjamé““

${ }^{6}$ Taxis communaux
} 
espace d'insertion dans un collectif et comme mode d'accès à une place dans la société (Safarti, 2015).

Cette étude repose essentiellement sur les théories de l'action, notamment les pratiques défendues par Castra (2004), qui ont pour objectif principal de replacer la personne "précaire » dans un environnement professionnel où elle pourrait redonner sens et consistance à son identité sociale.

L'objectif de ce travail est de montrer que les transports en commun à Abidjan ne sont pas seulement des espaces de violences et d'insécurité, mais qu'ils constituent aussi des espaces d'opportunités de travail et d'autonomisation et donc d'insertion sociale pour des jeunes à risque. Une telle approche pourrait manifestement agir sur la délinquance juvénile, en la modifiant positivement.

Cette approche suscite alors, un certain nombre de questions :

- quelles sont les opportunités d'emploi offertes par les transports en commun?

- quelles sont les caractéristiques des jeunes qui intègrent ces emplois?

- quelles sont les motivations et les attentes de ces derniers par rapport à ce secteur d'activité ?

- quel est l'impact de cette insertion professionnelle sur le développement de la délinquance juvénile ?

Les résultats de ce travail qui seront précédés de la méthodologie de l'étude, seront structurés autour des caractéristiques socio-économiques des jeunes, des opportunités d'emplois, de l'attractivité de ce secteur d'activité pour jeunes à "risque" et de son impact éventuel sur la délinquance juvénile.

\section{METHODOLOGIE}

\subsection{Site et participation à l'enquête}

L'étude s'est déroulée à Abidjan du 11 février au 17 mai 2017, soit un peu plus de trois (3) mois. Le choix du terrain d'étude a été motivé par le fait que le chômage en Côte d'Ivoire est essentiellement urbain et que $47 \%$ des jeunes au chômage vivent à Abidjan ${ }^{7}$.

Le choix spécifique du milieu des transports assuré par les "gbakas" et "wôrô-wôrôs" comme espace à investiguer a été guidé par le fait que ce secteur est devenu un espace de développement des corporations professionnelles et syndicales qui en ont fait de véritables places forces à la fois autonomes et

${ }^{7}$ Ministère de la promotion de la jeunesse de l'emploi des jeunes et du service civique (MPJEJSC) à travers Son document de politique nationale de la jeunesse et des stratégies 20162020 
revendicatives (Lombard, 2006) et où de nombreux jeunes en difficultés y travaillent.

$\mathrm{Au}$ cours de cette enquête de terrain, différentes catégories sociales susceptibles de rendre compte de l'objet ont été interrogés, à savoir des responsables et agents d'organisations syndicales, des chauffeurs, des apprentis, des chargeurs et des usagers. C'est en définitive 207 personnes, obtenues à partir d'un échantillon par jugement qui ont répondu à nos préoccupations à travers différentes communes de la ville d'Abidjan, notamment "Adjamé" (cette commune abrite la gare routière et le marché. C'est de là que partent les "gbakas" pour desservir les autres communes), "Abobo" (cette cité populaire est la plus peuplée de la ville d'Abidjan), "Yopougon" (cité populaire et la plus vaste de la ville d'Abidjan) et "Cocody" (commune résidentielle).

Pour les besoins de l'étude, nous avons retenu comme population cible, des jeunes en âge de travailler, tel que prévu dans le décret n $72-746$ du 24 novembre 1972 de la République de Côte d'Ivoire. La population d'enquête est donc composée de jeunes de 14 à 35 ans présents dans des activités au sein des transports en commun de la ville d'Abidjan. Le choix des unités statistiques s'est fait de manière empirique.

\subsection{Recueil et analyse des données}

Au niveau du recueil de données, les méthodes d'enquête utilisées ont été l'entretien et l'observation directe effectués par nous et deux (2) enquêteurs délégués.

Pour ce qui est de l'entretien, c'est un guide constitué de questions ouvertes qui a été proposé aux enquêtés, afin de permettre à ces derniers de s'exprimer assez librement sur leur vécu et les logiques qui sous-tendent leurs actions.

L'observation directe quant à elle, a permis d'intégrer le milieu des transports en commun par l'intermédiaire de certaines organisations syndicales pendant plusieurs jours à différents endroits. Cette approche permet de constater de visu les attitudes et comportements des individus dans le secteur des transports en commun.

Les données obtenues dans le cadre de cette enquête ont été organisées, traitées et analysées essentiellement au plan qualitatif avec quelques fois des descriptions quantitatives. Ce choix a été favorisé par l'absence de statistiques fiables et la volonté de recueillir le maximum de données d'opinion et de faits. 


\section{RESULTATS}

\subsection{Caractéristiques socio-économiques des jeunes}

Il s'agit dans cette partie du travail de décrire les caractéristiques des jeunes en activité, pour connaître leur répartition par tranche d'âge, lieu d'habitation et niveau d'étude.

Tableau 1 : Distribution des enquêtés selon la tranche d'âge

\begin{tabular}{|c|c|c|}
\hline Tranche d'âge (ans) & Effectifs des enquêtés & Pourcentages (\%) \\
\hline$[14-18[$ & 32 & 22,07 \\
\hline$[18-25[$ & 64 & 44,14 \\
\hline$[25-30[$ & 26 & 17,93 \\
\hline$[30-35]$ & 23 & 15,86 \\
\hline Total & $\mathbf{1 4 5}$ & $\mathbf{1 0 0}$ \\
\hline
\end{tabular}

Source : enquête de terrain

Les résultats du tableau 1 montrent que $22,07 \%$ des jeunes interrogés ont un âge compris entre 14 et 18 ans, tandis que $44.14 \%$ ont un âge qui varie entre 18 et 25 ans. On constate également que $17.93 \%$ de ces acteurs ont un âge compris entre 25 et 30 ans. Enfin, ceux dont l'âge se situe entre 30 et 35 ans représentent 15,86 de la population cible.

Ce tableau indique que les jeunes en activité dans le secteur des transports en commun sont ceux dont l'âge se situe entre 18 et 25 ans, suivis des 14 à 18 ans, ensuite les 25-30 ans et enfin les 30 à 35 ans. Cela témoigne de ce que les plus jeunes [14-25[sont de loin les plus nombreux avec 66,21\% contre $33,79 \%$ pour les moins jeunes [25-35], soit presque le double.

Tableau 2 : Distribution des enquêtés selon le lieu d'habitation

\begin{tabular}{|c|c|c|}
\hline Lieu d'habitation & Effectifs & Pourcentages (\%) \\
\hline Quartier précaire & 116 & 80 \\
\hline Quartier résidentiel & 00 & 00 \\
\hline Quartier à standing moyen & 11 & 7,59 \\
\hline Au sein des gares & 18 & 12,41 \\
\hline Total & $\mathbf{1 4 5}$ & $\mathbf{1 0 0}$ \\
\hline
\end{tabular}

Source : enquête de terrain

Les résultats du tableau 2 montrent que $80 \%$ des enquêtés habitent des quartiers précaires, tandis que 7,59\% d'entre eux disent résider dans des quartiers à standing moyen. Il ressort également des données du tableau qu'aucun des sujets interrogés n'habite dans une cité de haut standing. 12,41\% de ces individus sont sans abris (dorment au sein des gares).

L'analyse de ce tableau permet de comprendre que les jeunes en activité dans le secteur des transports en commun sont en grande majorité issus des quartiers défavorisés ou précaires. 
Tableau 3 : Distribution des enquêtés selon leur niveau d'études

\begin{tabular}{|c|c|c|}
\hline Niveau d'instruction & Effectifs & Pourcentages \\
\hline Analphabète & 91 & 62,76 \\
\hline Primaire & 30 & 20,69 \\
\hline Secondaire & 17 & 11,72 \\
\hline Supérieur & 07 & 4,83 \\
\hline Total & $\mathbf{1 4 5}$ & $\mathbf{1 0 0}$ \\
\hline
\end{tabular}

Source : enquête de terrain

Les résultats du tableau 3 montrent que 32,41\% des jeunes interrogés affirment avoir fait le primaire et le secondaire tandis que $4,83 \%$ attestent avoir fait des études supérieures à l'université et dans des grandes écoles. 62,76 de la population cible, soit plus de la moitié affirme n'avoir pas été à l'école et par conséquent ne sait ni lire, ni écrire. Nous pouvons affirmer en observant les résultats de ce tableau que la plupart des jeunes en activité dans le secteur des transports en commun sont analphabètes.

Les recherches effectuées n'ont pas permis au plan statistique de connaître avec exactitude le nombre des jeunes en difficultés en activité dans le secteur des transports en commun à Abidjan. Leur nombre est inconnu des pouvoirs publics et mal connu des organisations syndicales en charge du milieu. Toujours est-il que ces jeunes sont en nombre important. Ce sont des milliers d'individus de divers horizons qui sont en activité dans le secteur des transports en commun. Ils sont pour la plupart des victimes de conditions familiales délicates, d'analphabétisme, de déscolarisation et sans qualification. Ce sont donc des catégories sociales en difficulté, en situation de vulnérabilité et donc à risque.

\subsection{Description des emplois les plus usités par les jeunes}

Le secteur des transports en commun de la ville d'Abidjan est un espace qui offre des possibilités d'emplois à tous ces jeunes sans distinction d'âge, de niveau d'étude et d'origine sociale. La chance est donnée à tous, d'avoir un boulot sans autre forme de prescription. L'essentiel, c'est de vouloir travailler et de pouvoir le faire. Les emplois les plus usités sont ceux de chauffeurs, d'apprentis et de chargeurs.

\subsubsection{Apprentis "gbakas"}

Ce sont des jeunes gens qui se chargent d'encaisser le titre de transport auprès des passagers à bord des "gbakas". Ils sont aussi chargés de signaler au chauffeur les descentes et les montées des passagers. Leur tâche consiste donc à aider le chauffeur à mener à bien son activité. Leur âge se situe en général entre 14 et 18 ans. Mais, il arrive que des jeunes plus âgés ou moins âgés (10 à 14 ans) s'adonnent à cette activité. 
Pour ce qui est de la rémunération, il faut noter que ces jeunes touchent entre 2000 et 3000 francs $\mathrm{CFA}^{8}$ par jour soit une rétribution mensuelle de 60.000 à 90.000 francs CFA.

\subsubsection{Chauffeurs}

Ce sont des jeunes qui en principe sont détenteurs d'un permis de conduire à qui on confie un véhicule pour le transport des usagers d'un point à un autre moyennant une rétribution. Ils sont donc pour la plupart des majeurs c'est-à-dire qu'ils n'ont pas moins de 21 ans. Ces chauffeurs doivent verser quotidiennement en fin de journée, une certaine somme d'argent dont le montant est convenu d'avance avec le propriétaire du véhicule ou le gérant. Les chauffeurs perçoivent mensuellement 50000 à 60000 Francs CFA.

\subsubsection{Chargeurs}

Il s'agit ici de personnes qui ont pour activité le chargement des véhicules de transports. Ils s'occupent également de l'organisation et de la discipline dans les gares et autres points de chargements des passagers. On retrouve dans cette catégorie toutes les tranches d'âges, c'est-à-dire de 14 à 35 ans et c'est l'activité qui emploie le plus de jeunes. Néanmoins, la présence de personnes plus âgées est constatée et celle des plus jeunes y est tolérée.

Les chargeurs en fonction des tâches qui sont les leurs, perçoivent une rétribution financière, allant de 2000 à 50000 Francs CFA par jour et quelques fois même un peu plus. Ces individus perçoivent alors un salaire mensuel qui part de 60000 à 1500000 Francs CFA.

Les emplois les plus usités dans le milieu des transports en commun à Abidjan sont ceux de chauffeurs, d'apprentis et de chargeurs. Ils permettent à de nombreux jeunes en situation de précarité, de vulnérabilité de gagner de l'argent et sûrement envisager un projet de vie.

\subsection{Raisons d'une insertion des jeunes à risque par le secteur des transports en commun}

Il s'agit ici, de montrer les motivations qui amènent des jeunes à risque à se diriger essentiellement vers le milieu des transports en commun pour avoir du travail et s'insérer.

\footnotetext{
${ }^{8} \mathrm{CFA}$ : franc des colonies françaises d'Afrique. 1 Euro $=653,77 \mathrm{~F} \mathrm{CFA}$
} 
$\underline{\text { Tableau } 4}$ : Distribution des jeunes selon leurs motivations pour le secteur des transports en commun

\begin{tabular}{|c|c|c|}
\hline Motivations & Effectifs & Pourcentages \\
\hline Rentabilité financière & 64 & 44,14 \\
\hline Absence de qualification & 39 & 26,9 \\
\hline Liberté d'action & 31 & 21,38 \\
\hline Autres & 11 & 7,58 \\
\hline Total & $\mathbf{1 4 5}$ & $\mathbf{1 0 0}$ \\
\hline
\end{tabular}

Source : enquête de terrain

Le milieu des transports en commun de la ville d'Abidjan se révèle attractif pour diverses raisons. En effet, le tableau 4 montre que $44,14 \%$ des jeunes interrogés regagnent le secteur des transports pour la rentabilité financière des activités disponibles, quand $26,9 \%$ intègrent ces activités par manque de qualification. Par ailleurs, $21,38 \%$ des jeunes y vont parce qu'ils disent se sentir en liberté et à l'aise, tandis que 7,58\% y sont pour diverses autres raisons. On constate que ce sont essentiellement la rentabilité financière, le manque de qualifications et la liberté d'action qui motivent la présence des jeunes à risque dans le secteur des transports en commun. Le choix de ces individus est guidé prioritairement par l'activité économique qui leur permet de se prendre en charge et de soutenir la famille (nourriture, santé et scolarisation des frères et sœurs). Dans une telle perspective, les jeunes interrogés affirment de façon unanime, ne pas voir l'opportunité d'une formation professionnelle, qui selon eux viendrait leur faire perdre du temps et de l'argent. À ce propos, un enquêté affirme : « moi, ce n'est pas formation qui m'intéresse. Je n'en ai d'ailleurs pas besoin, car c'est une perte de temps. Je dis que je ne suis pas allé à l'école et vous me parlez de formation. Quelle formation? Pour m'installer où, avec quels moyens et quelle clientèle ?formation ou pas formation, le plus important, c'est l'argent pour manger et faire vivre la famille qui fait courir (préoccupe) tout le monde ».

Comme on peut le constater, les jeunes à risque échappent certes au cadre normatif ou formel, sélectif et exclusif de l'emploi, mais leurs préoccupations est autre. L'essentiel pour ces individus, c'est de gagner de l'argent immédiatement pour faire face à leurs besoins, et cette opportunité ils l'ont avec les transports en commun qui permettent de gagner d'importantes sommes d'argent. En effet, un chauffeur par exemple, en plus de son salaire mensuel qui est de 50000 à 60000 francs CFA fait un gain parallèle de 10000 à 15000 francs CFA par jour, soit 300000 à 450000 francs CFA par mois. Il en est de même pour les apprentis qui, indépendamment du salaire mensuel qui est de 60000 à 90000 francs CFA, font des gains parallèles d'environ 2000 à 3000 francs CFA par jour soit 60000 à 90000 francs CFA par mois. Les chargeurs connaissent eux aussi des gains parallèles qui varient de 2000 à 50000 francs CFA en fonction de leurs responsabilités. 
En termes de cumul de gains mensuel, un chauffeur peut se retrouver avec la somme de 350000 à 500000 francs CFA, quand l'apprenti lui gagne une somme qui oscille entre 120000 et 180000 francs CFA. Pour ce qui est des chargeurs, ils se retrouvent en cumul de gain mensuel avec une somme de 120000 à 3000000 de francs CFA en fonction des responsabilités qui sont les leurs.

Le milieu des transports en commun se révèle alors être un pôle attractif majeur, car d'importantes sommes d'argent y sont disponibles et offrent à de nombreux jeunes à risque, des opportunités d'existence, d'autonomisation et d'insertion sociale, comme en témoigne les propos suivants :

" Grand frère, il y a de l'argent dans travail de la gare et ce que je gagne ici me permet de payer ma maison et mettre mes frères à l'école. J'ai même une copine de qui je m'occupe convenablement maintenant" (K.S, Apprenti "gbaka').

«Je vis avec ma femme et ma fille que j'ai inscrit dans une école privée, alors que moi-même je ne suis jamais allé à l'école. C'est donc l'argent que je gagne à la gare ici qui me permet de faire tout cela. Sinon avant j'étais un voyou, un bagarreur et rejeté par tous.

Aujourd'hui je suis respecté en famille » (B.S, chargeur).

Le dynamisme économique et financier du secteur des transports en commun, en fait un secteur essentiellement attractif pour ces jeunes en quête d'insertion sociale.

L'attraction des jeunes par rapport au milieu des transports en commun s'expliquent aussi par la liberté d'action. En effet, les enquêtés ont révélés choisir le secteur des transports en commun pour se débrouiller, car dans cet espace, ils sont libres d'agir pour gagner de l'argent. Ce contexte, ils disent ne pas le retrouver en milieu fermé, notamment dans les centres de formations et autres établissements d'apprentissages, où ils sont souvent à la merci des patrons, qui les considèrent comme des esclaves. Les transports en commun constituent alors pour eux, un espace d'épanouissement social et professionnel, contrairement aux centres fermés d'apprentissages qui se comportent à leurs yeux comme des prisons, c'est-à-dire un espace à mobilité (horizontale et verticale) réduite.

\subsection{Abandon de pratiques délinquantes au profit de comportements pro- sociaux}

Il s'agit de montrer dans cette partie du travail que les activités économiques menées, ont permis à de nombreux jeunes à risque d'abandonner les pratiques délinquantes au profit de comportements pro-sociaux. 
Tableau 5 : Distribution des jeunes selon leurs activités délinquantes antérieures

\begin{tabular}{|c|c|c|}
\hline Motivations & Effectifs & Pourcentages \\
\hline Bagarres & 67 & 46,21 \\
\hline Vols & 53 & 36,55 \\
\hline Braquages & 17 & 11,72 \\
\hline Autres & 8 & 5,52 \\
\hline Total & $\mathbf{1 4 5}$ & $\mathbf{1 0 0}$ \\
\hline
\end{tabular}

Source : enquête de terrain

Le tableau 5 montre que 46,21\% des enquêtés avaient la bagarre comme activité principale par le passé, quand 36\% eux ont affirmé qu'ils vivaient de vols. $17 \%$ de notre population cible a avoué avoir participé à des braquages, qui constituaient l'essentiel de leurs activités. C'est seulement $8 \%$ qui disent s'adonner par le passé à d'autres activités délinquantes sans grande importance, telles que les jeux de hasard et autres délinquances mineures. La consommation de la drogue est transversale chez ces jeunes gens.

La bagarre qui apparaît comme la chose la mieux partagée dans les rues d'Abidjan, selon certains propos, permettait à certaines personnes de se faire craindre et aimer. Cette fonction de grands bagarreurs aux dires des enquêtés suffisait pour obtenir de la part des autres tout ce qu'on veut comme argent ou autres biens matériels. Pour ce qui est du vol, l'activité consistait à arracher les sacs à main et les chaines des dames. Il consistait aussi à s'introduire frauduleusement dans des domiciles ou boutiques de commerçants pour dérober de l'argent ou des marchandises. La troisième activité délinquance majeure est le phénomène des braquages de véhicules, de domiciles et boutiques commerciales. Cette catégorie de personnes paraît la plus dangereuse, car ils disent qu'ils agissaient avec des armes à feu et par conséquent, ils étaient souvent auteurs de criminalité violente.

Ces trois activités sus mentionnées, représentent plus de 94\% des activités criminelles que ces jeunes gens disent avoir abandonné pour se consacrer essentiellement aux activités économiques "licites" que leur offre le secteur des transports en commun. Ces activités, en plus de leur permettre de gagner de l'argent et de vivre correctement selon leurs dires, leurs dispensent des angoisses et craintes de se faire arrêter ou abattre par la Police. Ils se consacrent désormais à leur travail et leurs familles. Ils sont même fiers d'avoir fondé une famille pour la plupart, qui les occupe utilement et leur apporte la joie. Ils ont avoué se sentir plus responsables et se situer dans une perspective d'aller de l'avant. On peut donc dire que le lien social qui avait été distendu est reconstruit et tout le corps social s'en porte mieux.

Les opportunités d'emplois qu'offrent les transports en commun permettent aux jeunes à risque de vraiment s'éloigner de la délinquance pour adopter des comportements pro-sociaux. Ils y gagnent "modestement" leur vie 
et son respectueux de la vie et des biens d'autrui. Un tel comportement contribue fortement à réduire les braquages, les vols et les violences et par conséquent à agir positivement sur la délinquance juvénile.

L'insertion sociale des jeunes en difficulté ou à risque à travers le secteur des transports en commun devra donc faire l'objet de partage d'expérience. Cette approche en permettant à de nombreux jeunes sans qualifications et souvent à la marge, de gagner de l'argent, d'être autonome et de s'insérer socialement contribue fortement à les extraire du cercle vicieux de la délinquance. Cette intégration sert d'ailleurs de modèle à d'autres jeunes en situation de vulnérabilité, qui intègrent le secteur des transports en commun, qu'ils perçoivent finalement comme une voie honorable pour sortir de la délinquance ou l'éviter.

\section{CONCLUSION}

Le milieu des transports en commun essentiellement perçu comme une niche de développement de l'insécurité urbaine, est également porteur d'opportunités d'emplois pouvant permettre de faire face au problème d'insertion sociale et professionnelle des jeunes en difficultés. En effet, ce bassin d'emplois précaires et informels, offert par le milieu des transports en commun, permet à ces jeunes pour la plupart analphabètes, déscolarisés, sans qualification ou encore issues de familles aux conditions délicates, de gagner "honnêtement" de l'argent et par conséquent d'exister, d'être autonomes et responsables. Ce secteur d'activité a aussi permis à de nombreux jeunes délinquants de mettre fin à leurs pratiques délinquantes qui leur permettaient de se prendre en charge, pour adopter une vie beaucoup plus décente. Il n'est donc pas prétentieux de dire que le secteur des transports en commun à Abidjan, par les opportunités d'insertions sociales qu'il offre contribue à réduire la délinquance des jeunes. C'est donc à juste titre que ce secteur d'activité constitue pour les jeunes, un pôle d'attraction majeur, car il permet une insertion par l'activité économique.

On gagnerait donc à s'investir d'avantage dans la compréhension de ce système d'intégration pour en faire un outil de réduction de la pauvreté et d'insertion sociale des jeunes sans qualification, vulnérables voire "précaires". Cela permettra à ces derniers, d'intégrer des projets prosociaux à même de leur offrir des perspectives d'intégration plus rassurantes et par conséquent atténuer les délinquances juvéniles.

\section{References:}

1. Castra, D. (2004) : L'insertion professionnelle des publics précaires. Presse universitaire de France, 2003 
2. CERTU. (2004): L'insécurité dans les transports publics urbains. Edition électronique consultée le 13 févier 2017 à 00 : 09 sur http://www.certu.fr.

3. Crizoa, H. (2010) : Les violences dans le milieu des transports en commun privés à Abidjan. Thèse unique de Doctorat de Criminologie, Option sociologie criminelle (non publiée), UFR criminologie, Abidjan, Université de Cocody, 230 p.

4. Crizoa, H. (2013) : Etudes des facteurs de résistance à l'interdiction de circulation des taxis banalisés (wôrô-wôrô intercommunaux) par les pouvoirs publics à Abidjan en Côte d'Ivoire. In Revue Africaine de Criminologie, numéro 13, p. 67-83.

5. Crizoa, H. (2014 a) : Violences 'utilitaires'” à Abidjan : le cas des Gnambrôs dans le milieu des transports en commun. In Revue Internationale de Recherches et d'Etudes Pluridisciplinaires, numéro 21, décembre 2014, $15 \mathrm{p}$.

6. Crizoa, H. (2014 b) : Conditions de travail et de vie des apprentis ' 'gbaka' et violences dans les transports en commun à Abidjan (Côte d'Ivoire). In Revue Africaine de Criminologie, numéro 14, p. 68-81.

7. Crizoa, H., Gbagbo M., \& Traoré, M. (2017) : femmes et frotteurisme dans les autobus à Abidjan. In Revue internationale de CRIMINOLOGIE et de POLICE Technique et scientifique.

8. Duchène, M \& Poplimont, C. (2013). «Insertion professionnelle, socialisation des jeunes français "sans qualification » et formation par alternance », Éducation et socialisation [En ligne], 34 | 2013, mis en ligne le 05 décembre 2013, consulté le 26 juin 2017. URL : http://edso.revues.org/460.

9. Garcia, (J). A. (2006) : Certaines choses communes que vous pouvez faire pour vous protéger vous-même. In Les nouvelles du Panama. Edition électronique consultéele 13févier 2017 à $02 \mathrm{~h} 13$ surhttp://www.thepanamanews.com/pn/v_12/issue_14/business_05.ht $\mathrm{ml}$

10. INHESJ/ONDRP. (2016): Les atteintes personnelles dans les transports en commun. "Cadre de vie et sécurité » de 2009 à 2013. La criminalité en France - In Rapport annuel de l'ONDRP.

11. Lombard, J. (2006). « Enjeux privés dans le transport public d'Abidjan et de Dakar », Géocarrefour [Online], Vol. 81/2 |2006, Online since 01 December 2009, connection on 22 June 2017. URL : http://geocarrefour.revues.org/1913; DOI : 10.4000/geocarrefour.1913.

12. Lombard, J. \& Ninot, O. (2002). « Impasses et défis dans le transport routier sénégalais ». In M. C. Diop (éd.). La société sénégalaise entre le local et le global. Paris : Karthala. p. 109-162. 
13. Ouimet, M \& Tremblay, P. (2001): Trajets urbains et risques de victimisation: les sites de transit et le cas du métro de Montréal. Criminologie. Vol.1, numéro 34. Edition électronique consultée le 13févier 2017 à $02 \mathrm{~h} 13$ sur http://doi.org/10.7202/004759r

14. Sarfati, F. (2015): "L'insertion professionnelle des jeunes entre précarité, incertitude et expérimentation " cahier de l'action, vol.45, $n^{\circ} 2, p p .9-16$.

15. Toumpsin, K. (2006) : Les transports en commun : espace d'insécurité ou de convivialité ? Bruxelles : Pax Christi. 